\title{
Premiando la mejor mazorca: AGRICULTURA Y EDUCACIÓN EN LA EsCUELA de Santa María de Dota en 1914
}

\begin{abstract}
Resumen
En el presente artículo se aborda la organización y premiación de la cosecha de la mejor mazorca de maíz en la escuela primaria de Santa María de Dota entre 1913 y 1914. Dentro del contexto de un espacio de colonización agraria, se resalta el interés por rescatar la motivación por parte de las autoridades encargadas de la agricultura en pro de mejorar los rendimientos de los cultivos. Por otro lado, se analiza la aplicación en la práctica agrícola de los conocimientos adquiridos en dicha localidad rural.
\end{abstract}

Palabras claves: agricultura, educación, rural, Santa María de Tarrazú, Costa Rica.

\begin{abstract}
The following article develops the topic of the organization and prize awarding of the best corn cob harvested in the elementary school of Santa María de Dota between 1913 and 1914. Within the context of the agrarian colonization, there is an important interest from the agricultural authorities in improving crop efficiency. Additionally, the article examines the agricultural application of rural, domestic cultivation knowledge.
\end{abstract}

Keywords: agriculture, education, rural, Santa María de Tarrazú, Costa Rica.

\section{Javier Agüero}

García. Egresado del Programa Latinoamericano de Doctorado en Educación, UCR. Magister Scientiae en Historia, UCR. Egresado de la Licenciatura en Docencia de la UNED. Excoordinador de la Sección de Historia y Geografía de la Sede de Occidente. Profesor de la Cátedra de Historia de la Cultura de la Escuela de Estudios Generales de la Sede Rodrigo Facio y de la Sección de Historia y Geografía del Departamento de Ciencias Sociales de la Sede de Occidente jav_aguero@hotmail.com

\section{Introducción}

Comúnmente se ha asumido que el saber aprendido en los centros educativos se queda nada más en los documentos, que día a día los estudiantes se actualizan con la información que les transmiten sus maestros. La distancia marcada entre el conocimiento almacenado en las notas escritas en cada hoja de los cuadernos y su práctica ha sido muchas veces objeto de las más severas críticas por parte de quienes abogan en favor de un modelo de educación más dinámico, susceptible de ser aplicado a lo largo de la vida.

La dinámica escolar debe ser matizada por el hallazgo de otro tipo de prácticas conducentes a una aplicación más inmediata de los conocimientos dictados por los maestros. En el presente artículo se explora lo acaecido en una escuela rural costarricense a principios del siglo pasado en el contexto de modernización 
agropecuaria costarricense. Esto, en razón de que en esos años hubo un interés del Departamento de Agricultura -encargado de promocionar e impulsar e cambio en las prácticas agrícolas - de poner a los estudiantes de las escuelas sembrar productos de primera necesidad. En este marco se organizaron concur sos que premiaban a quien cosechare la mazorca de maíz con mayor cantidad de granos.

El espacio por estudiar se ubica en la Escuela de Santa María de Dota, ubicada en el valle intermontano de Tarrazú, enclavado en el sureste de la provincia de San José. Como lo señala Hall (1984), su poblamiento, mediante procesos de colonizacion agricola espońánea, al igual que el de oros lugares del pais como Acosta y Puniscal, data del úl mo tercio del siglo XIX, durante los tiempos de la expansión de cultivo el cafe. A las tierras tarrazuceñas llegaron pioneros con sus

Debido al incremento poblacional y por la iniciativa de los vecinos, se erigió el cantón de Tarrazú en 1868, luego se fundaron las escuelas en cada uno de los unidos de Santa Maria, de San Marcos y de San Pablo, conformantes de dicha Marí porticiparon en el cer. Losen para la cosecha de la mazorca con mayor cantidad de granos y uno de ellos ganó el primer lugar en 1914 (Agüero, 2002). Los primeros pobladores, durante la segunda mitad del siglo XIX fundaron Los pris los pueblos de Santa Maria, de San Marcos y de San Pablo, a su paso abrieron subsistencias y luego, Juntozaron a comercializar los excedentes destinlivo de subsistencias $y$ l luego, empezaron a comercilizar los exceden destinados a la nant para sa ción en la agricultura, el cual también se dio en otros lugares del país como noroeste del Valle Central, en pueblos como Naranjo y Palmares (Samper, 1985). Al principio de la ocupación de los campos por parte de los inmigrantes, cultivo de los productos que aseguraban las por parte de los inmigrantes, el cultivo de los productos que aseguar las subsistemo máz y frijol: además de tubérculos y otros productos cosechados merced a la generosidad de las tierras recién deforestas (Hall 1984). Así, gracias al esfuerzo tesonero de las familias, se empezó a producir artículos que formaban parte de la dieta campesinamilias, yuca, aguacate y cucurbitáceas (v. g. ayotes y chayotes). El café llegó tiempo despaisaje rural.

\section{La producción de subsistencias en Tarrazú}

Como se anotó anteriormente, el cultivo de productos básicos para la subsistencia fue una tarea fundamental para las familias pobladoras de los frentes de colonización. El acto de voltear la montaña en Tarrazú supuso una serie de retos de mayor envergadura para los vecinos del noroeste del Valle Central (Naranjo Palmares y San Ramón), con áreas relativamente planas y con el aliciente de la conexión con el camino nacional vía Puntarenas (Hilje, 1992). En cambio, las ta conexión con el camino nacional via Puntarenas (Hilje, 1992). En cambio, las con una topografía escarpada que dificultaba la construcción de caminos; todos estos fueron aspectos adversos con los que los pioneros lidiaban cada día. E camino más antiguo conectaba la región con el sur de la ciudad capital dí. ficamente con la localidad de El Higuito; las carretas partían del caserío de San
Pablo, subían el alto del cerro Abejonal y, luego de más de veinte kilómetros, subían la cuesta de la montaña del Tablazo para, por último, entroncarse con Desamparados. Cerca de allí se ubicaba la finca, propiedad de la familia Ortuño, dueña de un importante beneficio llamado La Raya (Agüero, 2002).

Según los datos arrojados por el Censo Agrícola de 1913 (véase el cuadro 1), durante el inicio de la colonización hasta bien entrado el siglo XX, los campesinos sembraban productos como el arroz, la caña de azúcar, el frijol y el maiz. Este último fue el de mayor proporción de área cultivada, en tanto que el arroz ocupaba uno de los renglones más bajos en lo relativo a su cultivo.

Además de estas actividades, también se destacan las asociadas al café (con 470 hectareas cultivadas) y la ganadería porcina y vacuna. Esta última, sobre todo, requeria de pastos con una extensión de 4308 hectáreas para 1913 (Anua-
rios Estadisticos, 1913).

Cuadro 1

Tarrazú: área cultivada según cultivo (1913)

\begin{tabular}{|c|c|}
\hline Cultivo & Hectáreas \\
\hline Arroz & 16 \\
\hline Frijol & 112 \\
\hline Caña de azúcar & 175 \\
\hline \multicolumn{2}{|c|}{ Maíz } \\
\hline $\begin{array}{l}\text { Fuente: Elaboración propia a partir de Anuarios Estadísticos. (1913). } \\
\text { San José Tipoografía Nacional. }\end{array}$ \\
\hline
\end{tabular}

\section{Los quehaceres en una tierra de agricultores}

La preocupación de las autoridades gubernamentales por elevar los rendimientos agrícolas encontró su más fiel aliado en las publicaciones oficiales, en el caso del es-
tímulo de la caficultura durante el siglo del grano de oro (1840-1940). Se promocionaron prácticas modernas para ese entonces, divulgadas en órganos informatiovos como el Boletín de Agricultura, entre otros (Naranjo, 1997). En el caso de los cultivos como los granos basicos, tambien se publico informacion relativa al mejoramiento de la calidad y de la cantidad de las cosechas, de ahi que no fuera fortuito que el la participación del concurso para la obtención de la mejor mazorca de maíz por parte de los escolares.

Además de lo estimulado por las publicaciones -a las que, por cierto, no todos tenían acceso- se puede notar como en Tarrazú, donde se premió al cultivador de la mejor mazorca, el aprendizaje de los oficios ligados al campo se hacía predominantemente mediante tres vías (Agüero, 2002):

La cotidiana: A partir del ejemplo práctico del padre agricultor, que llevaba a su hijo al campo y, poco a poco, con el paso del tiempo, iniciaba faenas más simples como desherbar; luego continuaba con actividades más complejas que lo perfilaban potencialmente como todo un agricultor, para así proveer del sustento diario a su familia. 
La instrucción mediante charlas: El Departamento de Agricultura se dedicaba a brindar conferencias encaminadas al perfeccionamiento de las labores en las fincas (Villalobos, 2009). Por ejemplo, entre febrero y mayo de 1912 hubo ocho charlas, con una asistencia de 471 vecinos, sin contar a los escolares (Boletín de
Fomento, 1912, N. ${ }^{\circ}$ ).

Entre los contenidos impartidos en esas charlas se destacan: la selección adecuada de las semillas, el avistamiento de los terrenos para la siembra y el uso correcto de los abonos.

Las lecciones de agricultura, impartidas en los planteles de enseñanza, fueron las de mayor alcance divulgativo en torno a la mejora de las prácticas agrícolas (Villalobos, 2009). Los temas desarrollados eran similares a los de las charlas dirigidas a los adultos, su diferencia radicaba en el nivel, en este caso, inferior en relación con el grado cursado por los niños. En las escuelas, las juntas de educación se ocupaban, ente otras cosas, de proveer de un espacio para dichas practicas agricolas.

Eanta María, la Junta de Educación de San Marcos disponía acatar lo dispuesto por las autoridades, por medio de la asignación de un terreno para la agricultura, esto, a propósito del proyecto

se procedió a dar lectura del oficio N. 727 del 28 de julio de 1913 que decía:

... con indicaciones del señor Inspector de Escuelas las juntas deben proveer a los maestros de un lote de tierra para los ensayos agrícolas en donde los niños aprendan teórica y prácticamente los principios científicos de agricultura se decidió dedicar ese lote al fin indicado (Junta de Educación de San Marcos de Tarrazú, 1908-1924., f. 57).

De igual manera, estos entes administrativos de las escuelas tenían dentro de De igual sus quehaceres suplir a los maestros de agricultura del utillaje de labranza, con Educación de San Marcos se refería a una donación de fertilizantes:

Artículo III El señor presidente manifestó que el Departamento Nacional de agricultura obsequió quintaly maida de Educación de San Marcos de Tarrazú, 1908-1924., pp. 69-70).

\section{El certamen}

Organizado por las autoridades de agricultura en 1913, el Boletín de Fomento del año siguiente exaltó la industriosa labor de la Junta de Educación de Santa María en cuanto a su participación y a su éxito. Al respecto, la citada publicación divulgó dicho aporte realizado por este centro educativo: Muy especialmente llamamos la atención de las juntas de educación sobre los
concursos del maíz que se organizan entre escolares y que uno de ellos, la junta de Santa María de Dota ha llevado a la práctica con el mayor éxito (Boletín de Fomento, 1914, N. ${ }^{\circ}$ 3., p.174)
Como corolario de lo anterior, se hizo una excitativa en la divulgación de los magníficos resultados obtenidos con la mejor cosecha de la mazorca, para que pudieran entonces revertirse en mejoras sustantivas de las prácticas agrícolas ejercidas por el resto de los cultivadores, para asi cumplir con la meta de alcanzar mejores rendimientos, pues de acuerdo con las autoridades:

..Ya no se verá en aquellos lugares sembrar milpas con las peores mazorcas que no se pueden vender bien, o con granos sin seleccionar y es probable que mucosechas crecidas (Boletín de Fomento, 1914, N. 3., p. 176).

Cabe además señalar que, desde marzo de 1913, fue la misma junta de educación de Santa María quien directamente encomendó a sus docentes a fin de que:

Conociendo esta Junta que la agricultura es la principal fuente de riqueza de los costarricenses y que es deber patriótico fomentarla por cuantos medios estén a su alcance, con el fin de llevar al ánimo de los escolares las sugestiones, de la de señor Inspector de escuelas y conferencista achacón se acuerda:

I- Instar a los maestros de la escuela para que hagan la propaganda entre los alumnos a fin de que cultiven cada uno por separado en área de maíz (Boletín de Fomento, 1914, N. 3., p. 178).

Para dar seguimiento a dicho concurso, que exaltaba un precepto con la patria, los campos cultivados por los escolares eran visitados con periodicidad por los maestros, quienes, además de tomar nota acerca del progreso o avance de las plantas, también proporcionaban consejo acerca de los mejores procedimientos de los cultivos.

Además, se señalaba que la cosecha sería recolectada por los niños acompañados de dos agricultores honorables. Los progresos de granos usados como semilla- se guardarín granos usados como semilla- se guardarian en costales con el respectivo nombre, a fin de que se pudiera presentar las mazorcas en el próximo

El ganador resultó ser el estudiante Bernardo Ureña. Su fotografía y el diploma de nardo Urena. Su fotografia y el diploma de tín de Fomento. Así, se le dio divulgación al tín de Fomento. Asi, se le dio divulgación al triunfador y, a la vez, se hacia un llamado a los mariense, de cuarto grado, en mención. Pues mariense, de cuarto grado, en mención. Pues prácticas agrícolas exitosas conducentes a la obtención de la mejor calidad de los frutos.

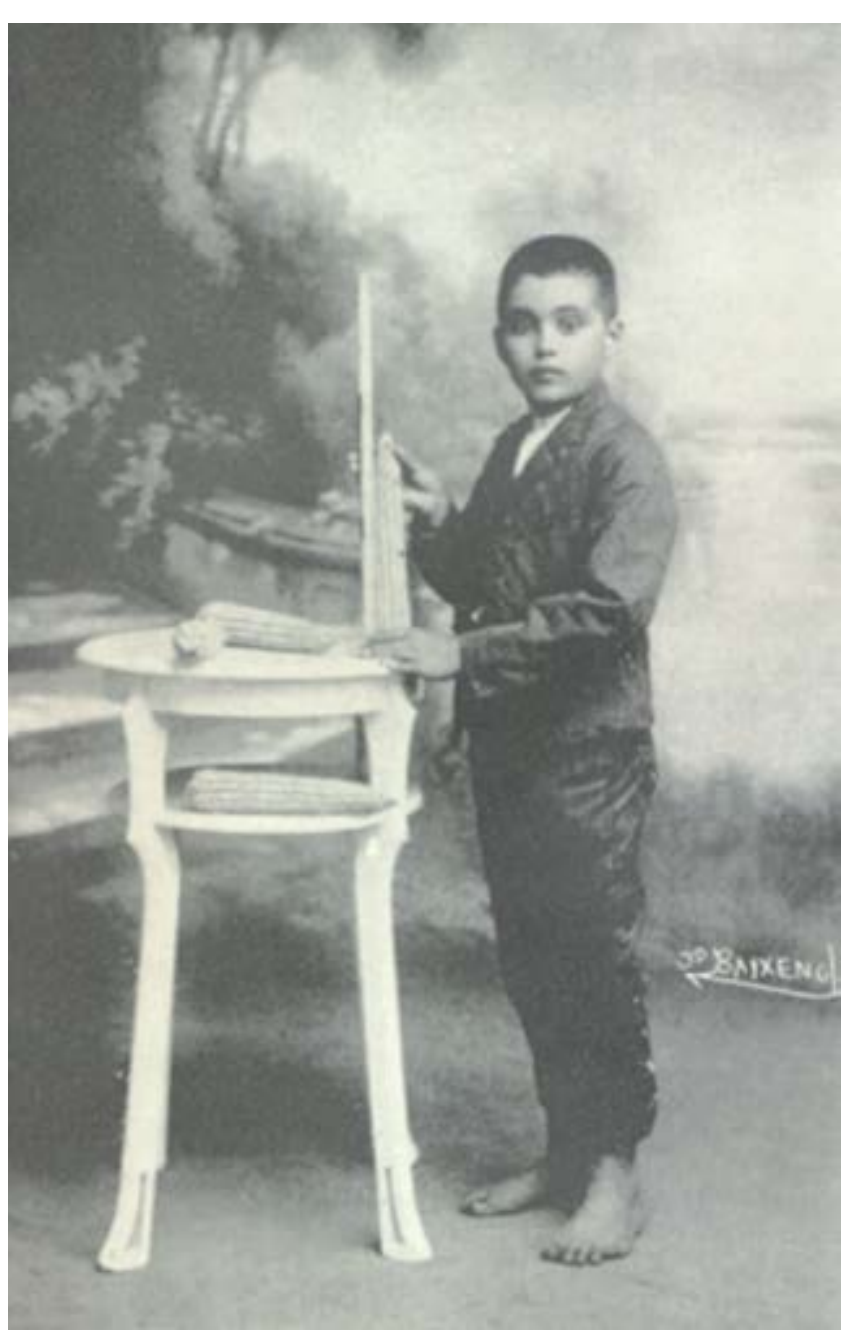




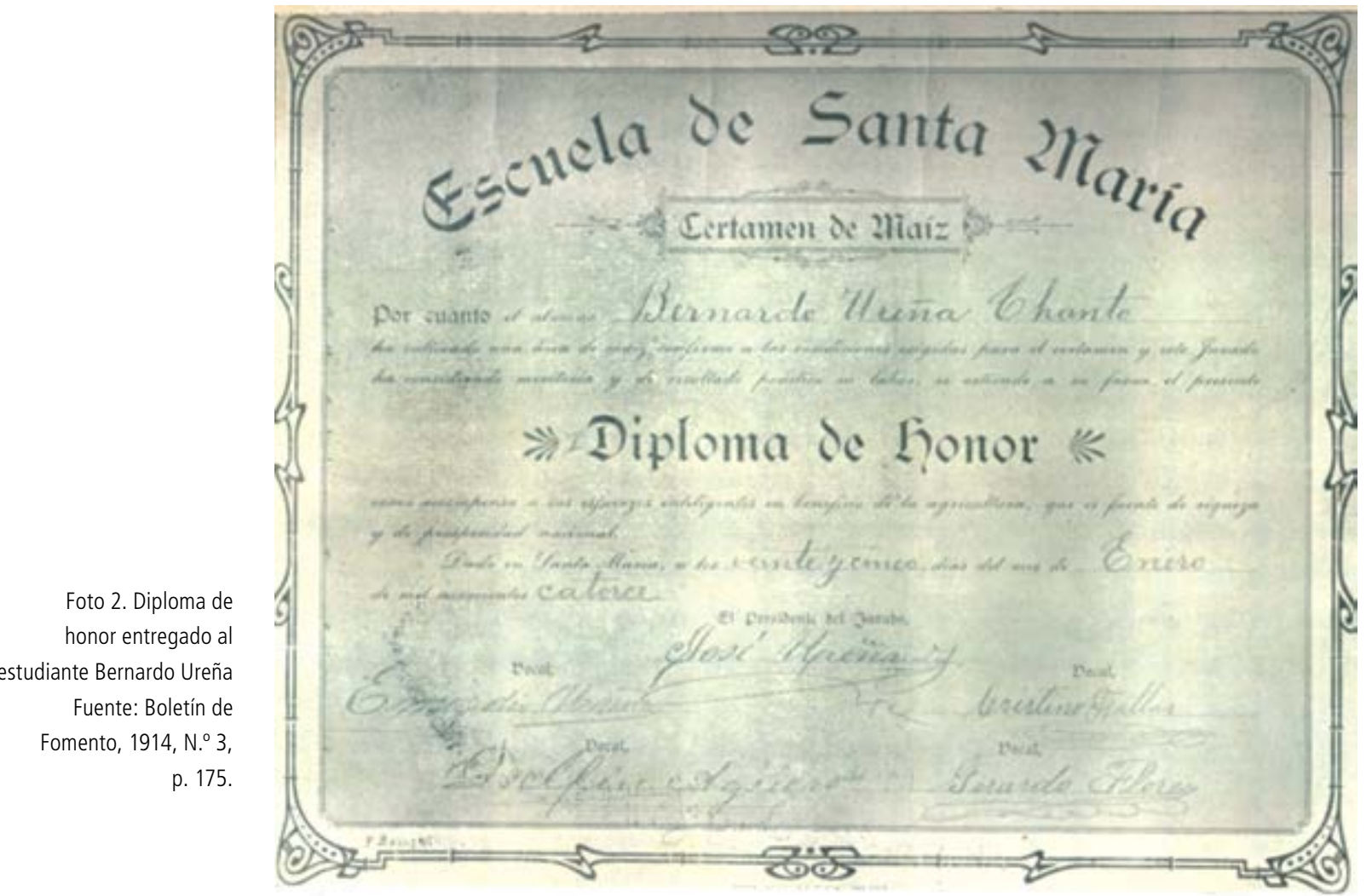

\section{Reflexiones finales}

En medio de las críticas más inmisericordes al sistema educativo que han cuestionado la institución desde sus mismas raíces, resalta el episodio estudiado en estas líneas porque propone una lectura alternativa acerca del ejercicio de la educación, por lo menos en el mundo rural costarricense.

La organización del concurso agrícola supuso una coordinación a lo interno del Departamento de Agricultura y también una adecuada comunicación con la comunidad educativa de Santa María. El objetivo primordial era aumentar las cosechas mediante la implementación de mejores prácticas agrícolas para asi aprovechar el insumo de los conocimientos agrícolas enseñados en los planteles educativos.

No cabe duda de que el ímpetu y la capacidad de sobresalir estuvieron presentes en la participación activa del centro educativo y del estudiante Ureña ganador del certamen. Esto, desde luego, pudo materializarse gracias al acompañamiento del personal del centro educativo, que motivó la participación para alcanzar el propósito: obtener la mazorca más grande.

La obtención de la cosecha de la mejor mazorca por parte de Bernardo Ureña tiene un doble significado. En primera instancia, la milpa era el referente contextual más inmediato de esta sociedad rural; algo así como lo visualizado por M. Samper cuando habla de un "paisaje social" relativo al tejido de relaciones resultantes de una dinámica productiva y organizativa alrededor de una práctica agrícola (1994). Pues esta geografía humana y, por tanto cultural, encarnaba un de las actividades más comunes en los pueblos productores de granos básicos. En un segundo plano, revela que las autoridades gubernamentales estaban interesadas en elevar los rendimientos agrícolas a partir del contacto con el mundo rural, de ahí que se premiara al cultivador de la mazorca de mayor tamaño.

Aunque, durante esa época, los modelos de las escuelas de aplicación de conocimientos - que habian sido toda una innovación en otras latitudes- no fueron desarrollados en el pais, es menester destacar que en el centro educativo de fuenanza primaria de santa Maria se puede encontrar un ejemplo de lo que fue llevar a la práctica los contenidos impartidos por los docentes. En este caso, merced a los cuidados de rutina aplicados a los campos, se logró cosechar una se presum por tanto, que los pueblos rurales no quedaru cultuador.

Se presume, por tanto, que los pueblos rurales no quedaron a la zaga en el pros y los libros; todo lo contrario, fueron el espacio idóntra par por los docentes y los libros; todo lo contrario, fuenon el espacio idóneo para preparar a los estudiantes para el mundo dé trabajo asociado a las labores agricolas, que en este medio camperi

La noción, tan arraigada en algunos textos responsables, de señalar la exclusividad del món décadas del siglo $X X$, fue caracterizado también por la producción le granos básicos y recibió el impulso dirigido hacia el aumento de la productividad del máz. En el caso de la organización y premiación del concurso en mención, se visualiza que el coctro agricola definitivamente iba más allá del cultivo del grano de oro. Por último en lo relativo al acontecer más próximo de quien escribe este texto. se puede indicar a guisa de reflexión final, que desde hace unos treinta años sepricultura dedicada a a im por las autoridades: solo interesa la promoción de los cultivos dedicados a la pxportación Casi ya no hay un lugar dentro de las prioridades oficiales para la producción nacional dedicada al autoconsumo o a la comercialización interna; produccón Castud de la puesta en rigor de las leyes del libre mercado, propugnadas por la políticas económicas neoliberales, que hicieron trizas la producción dedicada a cultivar lo que se come en el país. Es por esta razón que llama la atención el concurso realizado entre 1913 y 1914, pues es, ante todo, una muestra de las políticas agrícolas de la Costa Rica de hace cien años, tan diferentes a las de nuestros días. Este concurso es uno de los restos del patrimonio social - conformante, sin duda alguna, de una práctica cultural- que se debería rescatar para garantizar la vinculación del aprendizaje escolar acompañado con la práctica y al mismo tiempo, con el impulso a la sabia estrategia de producir lo que se consume.

\section{Bibliografía}

Fuentes primarias manuscritas

Junta de Educación de San Marcos de Tarrazú. Libro de actas (1908-1924).

Fuentes primarias publicadas

Anuarios Estadísticos. (1913). San José: Tipografía Nacional. 
Boletín de Fomento. (1912, N.²). San José: Tipografía Nacional.

Boletín de Fomento. (1914, N. 3). San José: Tipografía Nacional.

Tesis

Agüero, Javier. (2002). En busca de nuevas tierras: la colonización de una zona de frontera agrícola en el Valle de los Santos, 1870-1927. Tesis de Maestría en Historia, Sistema de Estudios de Posgrado. Universidad de Costa Rica.

Villalobos, Gabriela. (2009). "El progreso redentor". La Sociedad Nacional de Agricultura, el Estado Liberal y la modernización agropecuaria en Costa Rica. 1897-1914. Tesis de Maestría en Historia, Sistema de Estudios de Posgrado. Universidad de Costa Rica.

\section{Revistas}

Naranjo, Carlos. (1997). “La primera modernización de la caficultura costarricense". Revista de Historia. (36): 79-105, jul-dic.

Samper, Mario. (1985). "La especialización mercantil-campesina en el noroeste del Valle Central: 1850-1900: elementos micro-analíticos para un modelo". Revista de Historia: Historia, problemas y perspectivas agrarias de Costa Rica. (número especial): 49-91.

\section{Libros}

Hilje, Brunilda. (1992). La colonización agrícola de Costa Rica (1840-1940). San José: EUNED.

Hall, Carolyn. (1984). Costa Rica: una interpretación geográfica con perspectiva histórica. San José: ECR.

Sandner, Gehrard. (1962). La colonización agrícola de Costa Rica, v.1 San José: Instituto Geográfico Nacional.

Samper, Mario. (1994). "Los paisajes sociales del café: reflexiones comparadas". En: Héctor Pérez Brignoli y Mario Samper (comps.). Tierra, café y sociedad. San José: FLACSO. 